\title{
Evaluation of the feasibility of acetabular cup pre-determination in revision total hip arthroplasty via X-ray of the bone stock of the anterosuperior acetabulum
}

\author{
Jingwei Zhang ${ }^{1 \dagger}$, Keyu Kong ${ }^{1 \dagger}$, Yingjun $\mathrm{Chi}^{2+}$, Xiaoliang Liu' ${ }^{1}$, Yiming Zeng ${ }^{1 *}$ and Huiwu $\mathrm{Li}^{1{ }^{*}}$ (D)
}

\begin{abstract}
Purpose: This study was aimed to explore (1) location on AP pelvic X-ray that displayed bone stock in anterosuperior acetabulum; (2) whether X-ray could provide enough evidence to evaluate whether bone stock could provide support for acetabular cup; (3) criteria to determine whether anterosuperior bone stock could provide sufficient support for cup on X-ray.
\end{abstract}

Methods: Our study retrospectively collected 43 patients who underwent revision THA for cup loosening from 2014 to 2019. The position of anterosuperior acetabular bone stock was compared between X-ray and CT-based 3-D reconstruction. Seventy-millimeter acetabular cup was implanted simulatively to obtain the contact line between acetabular cup and superolateral remaining bone stock. The contact line length and the angle were measured. Patients were divided into cup group and cage group, and ROC curves of both contact line length and angle were drawn.

Results: The superolateral part of acetabulum on X-ray could reflect the anterosuperior host bone stock of acetabulum according to the comparison of anteroposterior pelvic X-ray and 3-D reconstruction. Critical point was chosen when we got the highest sensitivity with a $100 \%$ specificity in ROC curves. The critical values of contact length and angle were $15.58 \mathrm{~mm}$ and $25.5^{\circ}$.

Conclusions: Surgeons could assess the anterosuperior bone stock of acetabulum by AP pelvic X-ray to decide whether revision could be done merely using cup or need customized cage. Clinically, when contact line length was larger than $16 \mathrm{~mm}$ or contact angle was larger than $25.5^{\circ}$, adoption of cup could obtain primary stability in the revision surgery in most cases.

Keywords: Bone defect assessment, X-ray, Revision hip arthroplasty, Prosthesis selection

*Correspondence: huiwu1223@163.com; xyhz29@163.com

${ }^{\dagger}$ Jingwei Zhang, Keyu Kong, and Yingjun Chi have contributed equally to this work.

${ }^{1}$ Shanghai Key Laboratory of Orthopaedic Implants, Department of Orthopaedic Surgery, Shanghai Ninth People's Hospital, Shanghai

Jiaotong University School of Medicine, 639 Zhizaoju Road,

Shanghai 200011, People's Republic of China

Full list of author information is available at the end of the article

\section{Introduction}

Currently, the number of patients undergoing revision total hip arthroplasty (RTHA) is rapidly increasing $[1,2]$. Among those who undergo RTHA, bone defects, especially acetabular bone defects, are some of the most common but difficult problems that surgeons face [3-6]. Thus, assessment of acetabular bone defects is critical for surgical planning and preoperative preparation [7]. original author(s) and the source, provide a link to the Creative Commons licence, and indicate if changes were made. The images or other third party material in this article are included in the article's Creative Commons licence, unless indicated otherwise in a credit line to the material. If material is not included in the article's Creative Commons licence and your intended use is not permitted by statutory regulation or exceeds the permitted use, you will need to obtain permission directly from the copyright holder. To view a copy of this licence, visit http://creativecommons.org/licenses/by/4.0/. The Creative Commons Public Domain Dedication waiver (http://creativecommons.org/publicdomain/zero/1.0/) applies to the data made available in this article, unless otherwise stated in a credit line to the data. 
Pelvic anteroposterior radiography is essential for patients undergoing revision THA. In particular, the evaluation of acetabular bone defects and the host bone stock remains meaningful. Previously, there have been several reports regarding acetabular bone defect assessment and classification, such as the Paprosky classification [8] and AAOS classification [9]. However, similar studies have reported that these classifications remain unsatisfactory in terms of accuracy and reliability [10-16]. Hence, significant errors may occur when different surgeons use these methods. Moreover, this may have adverse effects on preoperative planning $[12,17]$. One possible reason for this is the difficulty in using a plain two-dimensional $\mathrm{X}$-ray to show the complex three-dimensional configuration and structure of the pelvis. Therefore, Choplin et al. [6] and Saleh et al. [17] proposed that anteroposterior (AP) pelvic X-rays could only provide a preliminary evaluation for RTHA; consequently, complex cases required further evaluation such as computed tomography (CT) and CT-generated three-dimensional models [18, 19]. Nevertheless, the role of primary assessment, the precise information that surgeons need to obtain from X-ray, and the significance of $\mathrm{X}$-ray results in pre-surgical planning are still unknown.

In RTHA, deciding whether using a cup alone can provide primary stability is critical in preoperative planning $[13,20,21]$. For surgeons, this may affect the surgical preparation and judgment of the difficulty of the surgery. Our previous study and clinical experience showed that the primary stability of the cup was achieved when the three-point support was distributed over the semicircle around the acetabulum. Mohamed et al. [13] introduced a new classification system for acetabular bone defects based on the three-point fixation theory. However, the precise locations of these three points are not illustrated in detail [13]. The rami ischii, rami ossis pubis, and the anterosuperior part of the acetabulum are anatomical structures that have the most bone stock and are most likely to have residual host bone left when bone defects occur around the acetabulum. If the host bone left in these three areas provides sufficient support for the cup, then the acetabular cup is sufficient on the acetabular side. According to our clinical experience, assessing rami ischii and rami ossis pubis based on AP pelvic radiography is relatively easy. The main problem is knowing whether the anterosuperior part of the acetabulum has enough host bone to support the cup, since this is not easily examined in AP pelvic radiography.

This retrospective study aimed to explore the following: (1) whether AP pelvic X-ray could be utilized to display host bone stock at the anterosuperior part of the acetabulum; (2) whether X-rays could provide sufficient evidence to evaluate whether the host bone stock at the anterosuperior part of the acetabulum could provide support for the cup; and (3) the criteria for determining whether the anterosuperior bone stock of the acetabulum could provide sufficient support for the cup based on the AP pelvic $\mathrm{X}$-ray results.

\section{Methods \\ Study participants}

This retrospective study was approved by the ethics committee of our institution. All patients who underwent RTHA for cup loosening between January 2014 and December 2019 were included in the study. Patients who refused to be involved in this study were excluded, whereas patients who underwent revision surgery due to infections and loosening of the femoral prosthesis that did not involve acetabular bone defects were also excluded. After the patients visited the hospital, radiographic evaluations of the pelvic and axial views of the affected hip joint were performed. Patients with mild bone defects (Paprosky [8]) were excluded. Finally, 43 patients with obvious acetabular bone defects were included in the study.

\section{Radiographic evaluation}

According to the selection of intraoperative prosthesis, the patients were divided into a cup group and a cage group. The cup group included patients who used the cup alone for revision, regardless of whether augment components were used. However, patients in whom screws were used to fix the augment or buttress plate in order to reconstruct the anterosuperior acetabulum were excluded. The cage group included patients who used cup-cage or customized cage for revision surgery. In the cage group, uncemented cups were unable to achieve sufficient fixation and stability after assessment during surgery. Thus, the cages were used for reconstruction. There were 34 and 9 patients in the cup and cage groups, respectively. Preoperative anteroposterior pelvic X-ray and preoperative hip joint thin-slice CT scan results of all patients in the two groups were collected. Three-dimensional reconstruction was performed based on the hip joint CT scan; thus, the influence of metal artifacts on CT was manually adjusted. Using the Medraw PrintV1 software, we matched the CT-based three-dimensional reconstruction model with the X-ray results. Calculations were made to obtain the two-dimensional projections of acetabular anterosuperior bone stock, which were selected in the three-dimensional model. The anterosuperior bone stock was defined as the area of anterosuperior acetabular bone mass thickening extending from the anterior inferior iliac spine. An example of this is shown in Fig. 1. 


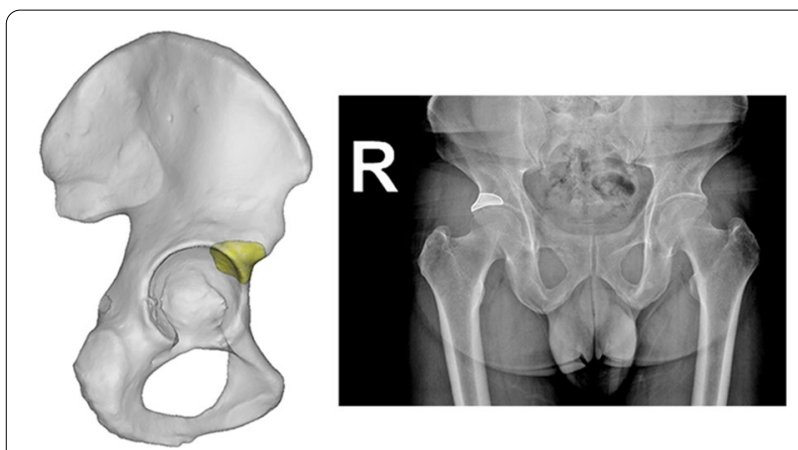

Fig. 1 X-ray of the anterosuperior bone stock of the acetabulum

Preoperative anteroposterior pelvic radiographs of all patients were obtained. We chose a 70-mm acetabular cup and simulated it in the patient's pelvis on radiographs. The 70-mm acetabular cup was the largest size we could obtain. If the 70-mm cup still could not fit the bone defect, the bone was unlikely to adopt the use of a cup in revision. The lower edge of the acetabular cup was determined using the lower edge of the acetabulum. Moreover, we simulated the cup with an abduction angle of $45^{\circ}$, which was the case in most patients. In addition, $45^{\circ}$ was optimal for cups in RTHA.

After determining the cup size, we determined the contact line between the cup and anterosuperior bone stock on the X-rays. We then measured the contact length and contact angle between the two contact ends and the rotation center of the hip joint (Fig. 2). To some extent, the contact line indicates the amount of contact between the anterosuperior part of the cup and the host bone, while the contact angle indicates the relative proportion of the anterior superior part of the cup to the host bone.

\section{Statistical analyses}

Statistical analyses were conducted using the Statistical Package for the Social Sciences (SPSS) version 17.0, for Windows (Inc., Chicago, IL). Receiver operating curves (ROC) were separately constructed using the length and angle of the contact line between the acetabular cup and anterosuperior bone stock, which were measured via $\mathrm{X}$-rays of the patients in the two groups (Fig. 3). The critical point was chosen when the sensitivity was the highest, with $100 \%$ specificity. According to our data, the critical contact length was $15.58 \mathrm{~mm}$, while the critical contact angle was $25.5^{\circ}$.

\section{Results}

The patients' demographic information and basic information of cups and cages are listed in Table 1, and the cup size of patients in the cup group is listed in Table 2. The anterosuperior host bone stock of the acetabulum

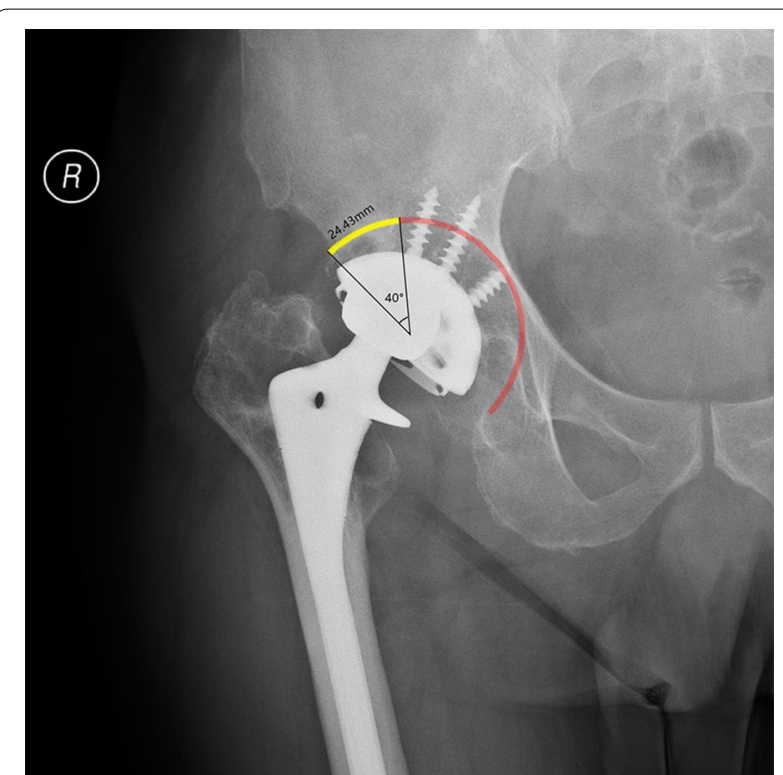

Fig. 2 Based on the simulative cup size and position, the contact length and angle between the acetabular cup and the superolateral part of bone stock on the two-dimensional X-ray image were measured

in the three-dimensional model is shown as the superolateral part of the acetabulum on the AP pelvic X-ray (Fig. 1).

$\mathrm{X}$-ray results could provide surgeons with sufficient information to determine the use of cups based on our

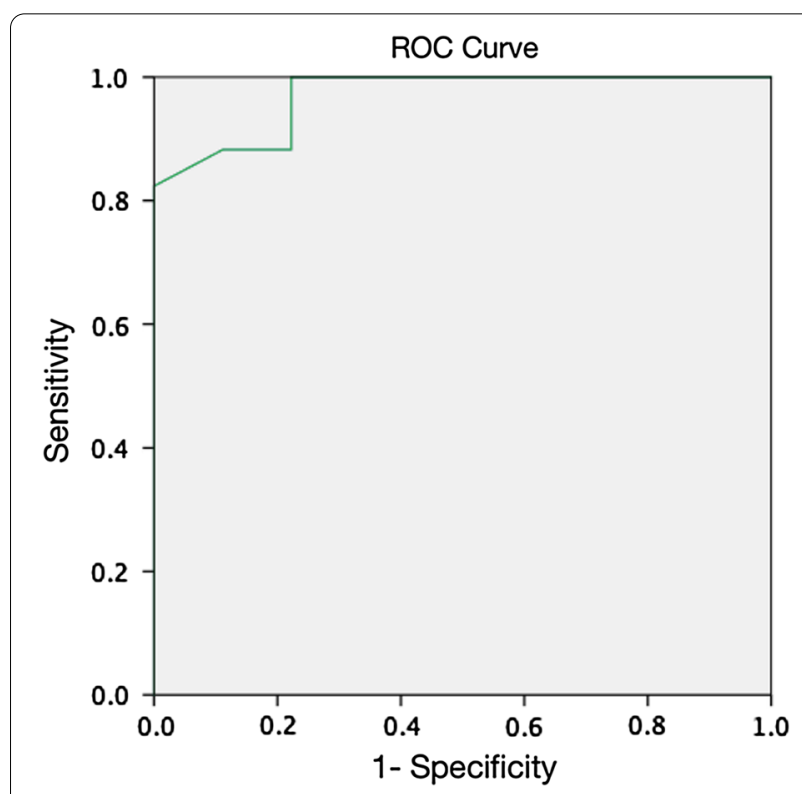

Fig. 3 Receiver operating curves (ROC) of the length and the angle of the contact line. The two curves overlap each other 
Table 1 Patient demographic background information

\begin{tabular}{llc}
\hline Demographic & Cup group & Cage group \\
\hline Gender, $n(\%)$ & & $6(66.7 \%)$ \\
Female & $22(64,7 \%)$ & $3(33.3 \%)$ \\
Male & $12(35.3 \%)$ & $67.47(30-92)$ \\
Age at surgery, mean (range) & $63.78(47-81)$ & $4 / 5$ \\
Site (left/right) & $17 / 17$ & Customized cage (9/9) \\
Type of cup/cage & Trabecular Metal Revision Shell (Zimmer Inc., Warsaw, Indiana, USA) (2/34) & \\
& Trabecular Metal modular acetabular system (Zimmer Inc., Warsaw, Indiana, USA) (29/34) & \\
\hline
\end{tabular}

Table 2 Sizes of cups used for patients in cup group

\begin{tabular}{ll}
\hline Size of cup & $\begin{array}{l}\text { Overall } \\
(\boldsymbol{n}=\mathbf{3 4})\end{array}$ \\
\hline 48 & 1 \\
50 & 5 \\
52 & 8 \\
54 & 1 \\
56 & 3 \\
58 & 3 \\
60 & 3 \\
62 & 3 \\
64 & 3 \\
66 & 3 \\
68 & 2 \\
70 & 1 \\
\hline
\end{tabular}

comparison of the contact line and the angle between the two groups. The ROC curve showed the critical point at which the highest sensitivity was achieved with $100 \%$ specificity. The critical contact angle was $25.5^{\circ}$, while the critical contact length was $15.58 \mathrm{~mm}$. As a result, we concluded that when the contact angle and contact length, measured using the aforementioned measuring method, were over $25.5^{\circ}$ and $15.58 \mathrm{~mm}$, respectively, an acetabular cup could be used as a primary revision prosthesis instead of a cup cage or a customized cage. Thus, considering its clinical application, a length of $16 \mathrm{~mm}$ is more convenient and practical in clinical practice.

\section{Discussion}

Acetabular bone defects are a major problem in RTHA $[3-6,10]$. In revision surgery, assessment of the bone defect via preoperative radiography is critical in deciding whether further evaluations, examinations, or extra preoperative preparations are needed. Moreover, the sufficiency of the cup alone for RTHA and knowing whether a customized prosthesis is needed for surgery are essential for preoperative preparation and surgical difficulty evaluation $[5,6,10,22,23]$.

In previous studies, there were several reports regarding acetabular bone defect assessment and classification, such as the Paprosky classification [8] and AAOS classification [9]. However, similar studies have shown that their accuracy and reliability are still unsatisfactory when conducting assessments [10-16]. However, it was difficult to assess relatively complex three-dimensional objects using simple two-dimensional images. In addition, the different training levels of doctors made these classifications less repeatable [12]. To assess complex bone defects through these classifications, considerable professional training is required. Currently, there are advanced evaluation methods, such as $\mathrm{CT}$ and rapid prototyping, which have been used for surgical planning and prosthesis fabrication for decades [24-28]. However, the need for three-dimensional information on bone defects from AP pelvic radiographs remains unknown. For the above reasons, several scholars believe that X-rays can only provide a rough evaluation $[6,17,29]$. However, the information provided by X-rays and the evaluation that can be made based on X-ray results remain unclear. Nevertheless, this does not mean that the $\mathrm{X}$-rays are not valuable. When performing radiological evaluations of patients who require hip revision, radiography is always the preferred initial examination. Meanwhile, it is very important to perform a primary assessment of the patient through radiography. In particular, for the surgeon, knowing whether the acetabular cup can be used for surgery or whether the cage or a customized prosthesis is needed is essential for preoperative preparation and judgment of the operation difficulty $[6,10,23,30,31]$. Therefore, we conducted this study to explore whether $\mathrm{X}$-rays could be used to determine whether patients could use cups for revision surgery.

In revision arthroplasty, we found that the premise for the reliable initial stability of the acetabular cup 
was whether the cup could acquire a three-point fixation within the boundaries of the acetabular distribution over $180^{\circ}$. The rationale was that only three-point clamping beyond the hemisphere could provide sufficient rotational stability. Ghanem et al. [13] also pointed out the importance of three-point fixation when faced with bone defects in revision arthroplasty in their study. However, the study did not specify the detailed requirements of the three-point distribution. Moreover, during the surgery, we found that the rami ischii, rami ossis pubis, and the anterosuperior and posterosuperior parts were the anatomical sites with the most abundant bone stock around the acetabulum. The posterosuperior part is the weight-bearing area of the acetabulum [32, 33]. Therefore, when a severe bone defect occurs around the acetabulum, it is generally the first to be damaged. Furthermore, it is difficult for the posterosuperior part to have an arrangement wherein one point is distributed more than $180^{\circ}$ relative to the other two. Therefore, the anterosuperior, rami ischii, and rami ossis pubis are the most likely anatomical sites to form a three-point effective fixation.

The bone stock of the rami ischii and rami ossis pubis is easily distinguished on X-rays, but the exact placement of the anterosuperior part of the acetabulum on $\mathrm{X}$-rays remains unknown. Moreover, there is no standard for determining whether the anterosuperior host bone stock can provide effective support when a bone defect occurs. Therefore, when bone defects occur, it is difficult to assess whether the anterosuperior host bone stock can provide effective support to form a three-point fixation. Therefore, in this study, an AP pelvic X-ray was calculated based on CT-generated three-dimensional models after the cup implantation procedure. We found that the superolateral part of the acetabulum in the AP pelvic $\mathrm{X}$-ray represented the anterosuperior part of the acetabulum in the 3-D model, which was consistent with our experience. This meant that we could assess the condition of the anterosuperior host bone stock of the acetabulum based on the AP pelvic X-ray.

This study also elucidated how to use the superolateral part of the acetabulum on X-ray imaging to determine whether the anterosuperior host bone stock of the acetabulum could provide sufficient support for the cup. In this study, we simulated cup implantation according to $\mathrm{X}$-rays. If the simulated implanted cup and the superolateral part of the acetabulum on X-ray could obtain a certain amount of direct contact, then during the operation, the anterosuperior host bone could provide sufficient support for the cup. The results of the ROC curve indicated that when the contact length was over $15.58 \mathrm{~mm}$ or when the contact angle was over $25.5^{\circ}$, the use of an acetabular cup would be sufficient for surgery. When implanting the cup, we first ensured that the lower edge of the cup was in contact with the expected rami ischii and rami ossis pubis. Second, we used an acetabular cup with a diameter of $70 \mathrm{~mm}$, the largest size available, since it could offer stable support for most patients. In clinical practice, surgeons can draw a $70 \mathrm{~mm}$ straight line with a $45^{\circ}$ angle from the horizontal line between the bottom of the acetabulum and the superolateral part of the acetabulum to simulate the diameter and abduction angle of a $70-\mathrm{mm}$ cup. In this study, a line with a $78.5^{\circ}$ angle from the simulated diameter of the cup was drawn, and the distance between the two intersections of this line and the superolateral acetabulum roughly represented the contact line according to our calculation. If the distance was over $16 \mathrm{~mm}$, the single use of the cup was sufficient for revision surgery because the real curved contact line was longer than the linear distance (Fig. 4). In this representative case, anterosuperior bone stock is sufficient to hold the cup with anteroinferior and posteroinferior bone stock. However, posterosuperior bone defect was found in preoperative evaluation and during operation which required an augment to assist the fixation of weight-bearing area.

Our study has several limitations. First, this was a retrospective cohort study; thus, we drew our conclusions after retrospectively comparing the host bone imaging on X-rays between the two groups, which were divided according to different intraoperative selections. However, the surgeon's choice of surgical approach, especially the use of cementless hemispherical/oval cup, cage/cup-cage, or customized prosthesis as the main prosthesis, is somewhat subjective and relies on the surgeon's discretion, which results in inevitable deviations. Therefore, high-quality prospective studies are required to verify the results of this study. Nonetheless, although several patients in the cage group could have used the acetabular cup, all patients in the cup group were verified intraoperatively to be eligible for cup use alone. Our selection method of the critical point guarantees that all patients satisfying the demands of the critical point could receive a revision THA with an acetabular cup. Second, the assessment of bone defects in several patients with RTHA is relatively difficult. If the surgeon suspected that the bone defect was serious, it was recommended to perform further evaluation via $\mathrm{CT}$, three-dimensional reconstructions, or rapid prototyping [24-28]. The X-ray assessment method used in this study was only intended to provide the primary assessment of the bone defect and to offer a reference for the selection of a surgical approach, which enables surgeons, especially doctors with less experience, to easily grasp the reference criterion when using $\mathrm{X}$-rays to conduct a primary assessment of patients. 


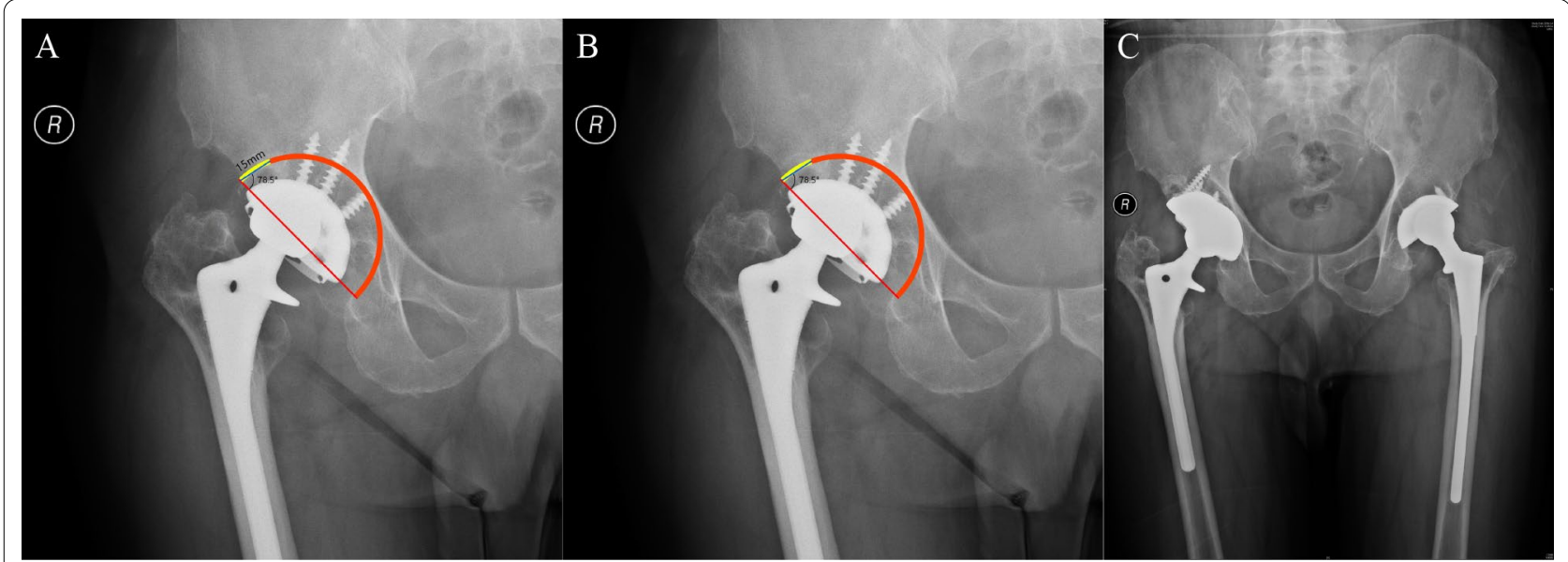

Fig. 4 Estimation of the contact length on X-rays in clinical practice. A The line with an angle of $78.5^{\circ}$ from the simulative diameter can best represent the contact curve. $\mathbf{B}$ Rough estimation of the contact line length is $15 \mathrm{~mm}$ in this patient, which means that he/she is capable of receiving a revision surgery with cup. C Postoperative pelvic AP X-rays of this patient

Third, this study did not conduct long-term follow-up of the included patients, and there were no reports on the long-term implant survival rate of revision joints. We plan to continue follow-up with these patients and report the follow-up results in due time. Finally, the number of patients included in this study was limited. Thus, we will attempt to enroll more cases in the future.

\section{Conclusions}

The results of this study show that the superolateral part of the acetabulum on AP pelvic radiographs can reflect the anterosuperior host bone stock of the acetabulum in patients who require RTHA. Based on whether the rami ischii and rami ossis pubis are intact, we can judge whether the acetabular cup can be used for revision surgery on the patient. At present, we propose the clinical criteria suggesting that the anterosuperior host bone stock of the acetabulum can provide sufficient support for the acetabular cup. Preoperative anteroposterior pelvic radiographs showed that the contact length of the simulated implanted cup and the superolateral host bone was greater than $16 \mathrm{~mm}$, and the angle between the lines connecting each end point of the contact line to the rotation center was greater than $25.5^{\circ}$.

\section{Acknowledgements \\ Not applicable.}

\section{Authors' contributions}

YZ and HL were responsible for the conception and design. HL provided administrative support. JZ and YZ provided the study materials and patients. $J Z$, KK, and YC collected and assembled data. JZ, KK, YC, and XL analyzed and interpreted the data. JZ, KK, and YC wrote the manuscript. All authors read and approved the final manuscript.

\section{Funding}

This study was sponsored by the National Natural Science Foundation of China (Grant No. 31900941) and the Interdisciplinary Program of Shanghai Jiao Tong University (Project No. ZH2018QNA06).

\section{Availability of data and material}

The datasets generated and/or analyzed during the current study are not publicly available because other studies are being carried out based on these data, but are available from the corresponding author upon reasonable request.

\section{Declarations}

\section{Ethics approval and consent to participate}

The study was conducted in accordance with the Declaration of Helsinki (as revised in 2013). The study was approved by the Ethics Committee of Shanghai Ninth People's Hospital affiliated to Shanghai Jiao Tong University School of Medicine, and informed consent was obtained from all participants.

\section{Consent for publication}

The authors affirm that the human research participants provided informed consent for publication.

\section{Competing Interests}

The authors declare that they have no competing interests.

\section{Author details}

'Shanghai Key Laboratory of Orthopaedic Implants, Department of Orthopaedic Surgery, Shanghai Ninth People's Hospital, Shanghai Jiaotong University School of Medicine, 639 Zhizaoju Road, Shanghai 200011, People's Republic of China. ${ }^{2}$ Department of Orthopedics, Shengzhou People's Hospital (the First Affiliated Hospital of Zhejiang University Shengzhou Branch), No.666, Dangui Road, Shengzhou 312400, Zhejiang, People's Republic of China.

Received: 21 June 2021 Accepted: 21 September 2021

Published online: 14 October 2021

\section{References}

1. Kurtz S, Ong K, Lau E, Mowat F, Halpern M. Projections of primary and revision hip and knee arthroplasty in the United States from 2005 to 2030. J Bone Joint Surg Am. 2007;89(4):780-5. 
2. Ong KL, Lau E, Suggs J, Kurtz SM, Manley MT. Risk of subsequent revision after primary and revision total joint arthroplasty. Clin Orthop Relat Res. 2010;468(11):3070-6.

3. Berry DJ, Lewallen DG, Hanssen AD, Cabanela ME. Pelvic discontinuity in revision total hip arthroplasty. J Bone Joint Surg Am. 1999;81(12):1692-702.

4. DeBoer DK, Christie MJ, Brinson MF, Morrison JC. Revision total hip arthroplasty for pelvic discontinuity. J Bone Joint Surg Am. 2007;89(4):835-40.

5. Rogers BA, Whittingham-Jones PM, Mitchell PA, Safir OA, Bircher MD, Gross AE. The reconstruction of periprosthetic pelvic discontinuity. J Arthroplasty. 2012;27(8):1499-506.e1.

6. Choplin RH, Henley CN, Edds EM, Capello W, Rankin JL, Buckwalter KA. Total hip arthroplasty in patients with bone deficiency of the acetabulum. Radiographics. 2008;28(3):771-86.

7. Ahmad AQ, Schwarzkopf R. Clinical evaluation and surgical options in acetabular reconstruction: a literature review. J Orthop. 2015;12:S238-43.

8. Paprosky WG, Perona PG, Lawrence JM. Acetabular defect classification and surgical reconstruction in revision arthroplasty. A 6-year follow-up evaluation. J Arthroplasty. 1994;9(1):33-44.

9. D'Antonio JA, Capello WN, Borden LS, Bargar WL, Bierbaum BF, Boettcher WG, et al. Classification and management of acetabular abnormalities in total hip arthroplasty. Clin Orthop Relat Res. 1989;243:126-37.

10. García-Cimbrelo E, García-Rey E. Bone defect determines acetabular revision surgery. Hip Int. 2014;24(Suppl 10):S33-6.

11. Käfer W, Fraitzl CR, Kinkel S, Puhl W, Kessler S. Analysis of validity and reliability of three radiographic classification systems for preoperative assessment of bone stock loss in revision total hip arthroplasty. Z Orthop Ihre Grenzgeb. 2004;142(1):33-9.

12. Campbell DG, Garbuz DS, Masri BA, Duncan CP. Reliability of acetabular bone defect classification systems in revision total hip arthroplasty. J Arthroplasty. 2001;16(1):83-6.

13. Ghanem M, Zajonz D, Heyde CE, Roth A. Acetabular defect classification and management: revision arthroplasty of the acetabular cup based on 3-point fixation. Orthopade. 2020;49(5):432-42.

14. Johanson NA, Driftmier KR, Cerynik DL, Stehman CC. Grading acetabular defects: the need for a universal and valid system. J Arthroplasty. 2010;25(3):425-31.

15. Gozzard C, Blom A, Taylor A, Smith E, Learmonth I. A comparison of the reliability and validity of bone stock loss classification systems used for revision hip surgery. J Arthroplasty. 2003;18(5):638-42.

16. Saleh KJ, Holtzman J, Gafni AL, Jaroszynski G, Wong P, Woodgate I, et al. Development, test reliability and validation of a classification for revision hip arthroplasty. J Orthop Res. 2001;19(1):50-6.

17. Saleh KJ, Holtzman J, Gafni A, Saleh L, Davis A, Resig S, et al. Reliability and intraoperative validity of preoperative assessment of standardized plain radiographs in predicting bone loss at revision hip surgery. J Bone Joint Surg Am. 2001;83(7):1040-6.

18. Munjal S, Leopold SS, Kornreich D, Shott S, Finn HA. CT-generated 3-dimensional models for complex acetabular reconstruction. J Arthroplasty. 2000;15(5):644-53.
19. John JF, Talbert RE, Taylor JK, Bargar WL. Use of acetabular models in planning complex acetabular reconstructions. J Arthroplasty. 1995:10(5):661-6.

20. Bürkner A, Fottner A, Lichtinger T, Teske W, Vogel T, Jansson $V$, et al. Primary stability of cementless threaded acetabular cups at first implantation and in the case of revision regarding micromotions as indicators. Biomed Tech (Berl). 2012;57(3):169-74.

21. Beckmann NA, Jaeger S, Janoszka MB, Klotz MC, Bruckner T, Bitsch RG. Comparison of the primary stability of a porous coated acetabular revision cup with a standard cup. J Arthroplasty. 2018;33(2):580-5.

22. Ballester Alfaro JJ, Sueiro FJ. Trabecular metal buttress augment and the trabecular metal cup-cage construct in revision hip arthroplasty for severe acetabular bone loss and pelvic discontinuity. Hip Int. 2010;20(Suppl 7):S119-27.

23. Li H, Qu X, Mao Y, Dai K, Zhu Z. Custom acetabular cages offer stable fixation and improved hip scores for revision THA with severe bone defects. Clin Orthop Relat Res. 2016;474(3):731-40.

24. Li H, Wang L, Mao Y, Wang Y, Dai K, Zhu Z. Revision of complex acetabular defects using cages with the aid of rapid prototyping. J Arthroplasty. 2013:28(10):1770-5.

25. Zerr J, ChatzinoffY, Chopra R, Estrera K, Chhabra A. Three-dimensional printing for preoperative planning of total hip arthroplasty revision: case report. Skeletal Radiol. 2016;45(10):1431-5.

26. McGurk M, Amis AA, Potamianos P, Goodger NM. Rapid prototyping techniques for anatomical modelling in medicine. Ann R Coll Surg Engl. 1997;79(3):169-74.

27. Wagner JD, Baack B, Brown GA, Kelly J. Rapid 3-dimensional prototyping for surgical repair of maxillofacial fractures: a technical note. J Oral Maxillofac Surg. 2004;62(7):898-901.

28. Harris J, Rimell J. Can rapid prototyping ever become a routine feature in general dental practice? Dent Update. 2002;29(10):482-6.

29. Garcia-Cimbrelo E, Tapia M, Martin-Hervas C. Multislice computed tomography for evaluating acetabular defects in revision THA. Clin Orthop Relat Res. 2007;463:138-43.

30. Chiang PP, Burke DW, Freiberg AA, Rubash HE. Osteolysis of the pelvis: evaluation and treatment. Clin Orthop Relat Res. 2003:417:164-74.

31. Noordin S, Masri BA, Duncan CP, Garbuz DS. Acetabular bone loss in revision total hip arthroplasty: principles and techniques. Instr Course Lect. 2010;59:27-36

32. Yoshida H, Faust A, Wilckens J, Kitagawa M, Fetto J, Chao EY. Threedimensional dynamic hip contact area and pressure distribution during activities of daily living. J Biomech. 2006:39(11):1996-2004.

33. Bergmann G, Deuretzbacher G, Heller M, Graichen F, Rohlmann A, Strauss $J$, et al. Hip contact forces and gait patterns from routine activities. J Biomech. 2001;34(7):859-71.

\section{Publisher's Note}

Springer Nature remains neutral with regard to jurisdictional claims in published maps and institutional affiliations.
Ready to submit your research? Choose BMC and benefit from:

- fast, convenient online submission

- thorough peer review by experienced researchers in your field

- rapid publication on acceptance

- support for research data, including large and complex data types

- gold Open Access which fosters wider collaboration and increased citations

- maximum visibility for your research: over 100M website views per year

At BMC, research is always in progress.

Learn more biomedcentral.com/submissions 